\title{
ECF Regimen
}

National Cancer Institute

\section{Source}

National Cancer Institute. ECF Regimen. NCI Thesaurus. Code C63389.

A regimen consisting of epirubicin, cisplatin and fluorouracil used alone or with radiation for the treatment of advanced-stage gastric cancer. 